\title{
基于微动磨损预测矿井提升钢丝绳安全系数
}

\author{
张 俊 $^{1}$ 葛世荣 $^{1,2}$ 王大刚 ${ }^{1,2}$ 张德坤 $^{3}$ \\ (1. 中国矿业大学机电工程学院 徐州 221116; \\ 2. 中国矿业大学江苏省矿山智能采掘装备协同创新中心 徐州 221116; \\ 3. 中国矿业大学材料科学与工程学院 徐州 221116)
}

\begin{abstract}
摘要: 为预测矿井提升过程中钢丝绳动态安全系数演变, 针对浅井低载荷矿井提升机, 运用动力学理论和摩擦传动理论获得 了距离容器不同位置处钢丝绳动张力, 建立了接触钢丝总磨损系数与钢丝间接触载荷、相对滑移和交叉角的关联模型, 提出 了矿井提升钢丝绳的磨损演化及承载安全系数预测方法, 探究不同工况参数对钢丝绳磨损及承载安全系数的影响规律。结果 表明: 随着提升循环次数的增加, 距容器不同位置处提升钢丝绳的横截面微动磨耗面积增大、安全系数降低; 钢丝绳悬垂中 间位置处, 提升钢丝绳承载安全系数下降较快; 提升钢丝绳承载安全系数对提升工况参数存在依赖性, 随着提升侧终端质量 的增加和最大提升速度的减小, 提升钢丝绳承载安全系数均呈降低趋势。
\end{abstract}

关键词: 矿井提升钢丝绳; 动态磨损; 安全系数; 微动磨损

中图分类号: TH117

\section{Prediction of the Safety Factor of Mine Hoisting Rope Based on Fretting Wear}

\begin{abstract}
ZHANG Jun $^{1}$ GE Shirong ${ }^{1,2}$ WANG Dagang ${ }^{1,2}$ ZHANG Dekun ${ }^{3}$
(1. School of Mechatronic Engineering, China University of Mining and Technology, Xuzhou 221116;

2. Jiangsu Collaborative Innovation Center of Intelligent Mining Equipment,

China University of Mining and Technology, Xuzhou 221116;

3. School of Materials Science and Engineering, China University of Mining and Technology, Xuzhou 221116)

Abstract: The evolution of dynamic safety factor of hoisting rope used in a shallow coal mine with the small terminal load is predicted in this study. Dynamic tensions of rope segments at different distances from the container are obtained employing kinetic theory and friction transmission theory. Correlation model between the total wear coefficients and contact load, relative slip and the crossing angle is established. Therefore, dynamic wear evolution and the safety factor of hoisting rope are calculated. Meanwhile, effects of parameters of working conditions on dynamic wear evolution and the dynamic safety factor of hoisting rope are explored. The results show that increases of hoisting cycles induce increased worn area of the cross-sections, and decreased safety factor of hoisting rope at distinct distances from the container. The dynamic safety factor of hoisting rope decreases rapidly in the middle location of vertical rope. The dynamic safety factor of hoisting rope correlates with hoisting parameters. An increase of terminal mass at the lifting side and a decrease of maximum hoisting speed are both induce decreased dynamic safety factor of hoisting rope.
\end{abstract}

Key words: mine hoisting rope; dynamic wear; safety factor; fretting wear

\section{0 前言}

提升钢丝绳连接着提升机和提升容器, 担负着 运输煤炭、生产设备和煤矿工作人员的任务, 其承 载强度和服役寿命直接关系到煤矿的正常生产和人

* 国家自然科学基金资助项目(51405489; 51375479)。20180419 收到初稿, 20180917 收到修改稿
员生命安全。在矿井提升过程中(上提和下放容器), 提升钢丝绳反复承受动张力作用，进而导致钢丝绳 (钢丝绳由股捻制而成, 而股由钢丝捻制而成)内部 钢丝间或股间摩擦磨损现象 ${ }^{[1]}$, 引起钢丝绳横截面 积降低, 使得提升钢丝绳承载强度和安全储备系数 (承载安全系数)产生变化。《煤矿安全规程》规定: 当钢丝绳直径缩小量或断丝数达到 $10 \%$ 时, 钢丝绳 应报废。但目前缺少定量评价提升钢丝绳运行过程 
中的磨损缩小量和安全系数动态演化的方法, 因而 无法预测提升钢丝绳的服役寿命和使用可靠性。

目前, 国内外研究学者构建了提升钢丝绳力学 模型 ${ }^{[2-4]}$, 研究了钢丝绳应力-应变关系, 计算了钢 丝绳内部钢丝拉力和钢丝间接触应力; 张德坤等 ${ }^{[5-11]}$ 研究了微动磨损过程中钢丝磨损机理和磨损深度演 化及其受到微动损伤参数和润滑油脂的影响; CRUZADO 等 ${ }^{[12-13]}$ 通过试验分析了接触载荷和交叉 角对钢丝微动磨损机理的影响; 王大刚等 ${ }^{[14]}$ 定量揭 示了不同环境介质下微动疲劳过程中钢丝的磨损深 度和磨损系数演化, 给出了基于断裂力学理论的钢 丝微动疲劳寿命预测模型。然而, 前人研究主要针 对钢丝绳内部钢丝的摩擦疲劳损伤研究, 并未将提 升钢丝绳动张力、钢丝绳内部钢丝或股磨损演化以 及提升钢丝绳承载安全系数进行关联研究。

本文选择一台浅井低载荷矿井提升机作为实 例, 研究接触钢丝总磨损系数与钢丝间接触载荷、 相对滑移和交叉角的关联模型, 建立矿井提升钢丝 绳的磨损演化及承载安全系数预测方法, 探究不同 工况参数对钢丝绳磨损及承载安全系数的影响规 律。所研究的提升系统及钢丝绳参数见表 $1^{[15]}$ 。

\section{表 1 钢丝绳及提升系统参数}

\begin{tabular}{lc}
\hline \multicolumn{1}{c}{ 提升钢丝绳型号 } & $6 \times 19+\mathrm{IWS}$ \\
\hline 钢丝绳弹性模量 $/ \mathrm{MPa}$ & $10^{5}$ \\
钢丝绳抗拉强度 $/ \mathrm{MPa}$ & 1570 \\
钢丝绳最小破断力总和 $/ \mathrm{N}$ & $1.19 \times 10^{6}$ \\
钢丝绳总横截面积 $/ \mathrm{mm}^{2}$ & 104.5 \\
钢丝绳每米质量 $/(\mathrm{kg} / \mathrm{m})$ & 0.975 \\
钢丝绳直径 $/ \mathrm{mm}$ & 16 \\
钢丝直径 $/ \mathrm{mm}$ & 1 \\
提升端终端质量 $/ \mathrm{kg}$ & 1400 \\
下放端终端质量 $/ \mathrm{kg}$ & 1000 \\
钢丝绳最大悬垂长度 $/ \mathrm{m}$ & 220 \\
提升行程 $/ \mathrm{m}$ & 200 \\
提升周期 $/ \mathrm{s}$ & 46.67 \\
加(减)速度 $/\left(\mathrm{m} / \mathrm{s}^{2}\right)$ & \pm 0.75 \\
最大提升速度 $/(\mathrm{m} / \mathrm{s})$ & 5 \\
\hline
\end{tabular}

\section{1 提升钢丝绳微动磨损参数计算}

\section{1 提升钢丝绳动张力}

简化的提升系统如图 1 所示, 提升过程中上提 侧、下降侧与摩擦轮切点处钢丝绳动张力 $S_{1} 、 S_{2}$ 分 别为 ${ }^{[16-17]}$

$$
\begin{gathered}
\ddot{S}_{1}=[E \cdot A \cdot(g+a)- \\
\left.\left(\frac{E \cdot A}{m_{1}+\rho \cdot L_{1} / 3}+a\right) \cdot S_{1}-2 \cdot v \cdot \dot{S}_{1}\right) / L_{1}
\end{gathered}
$$

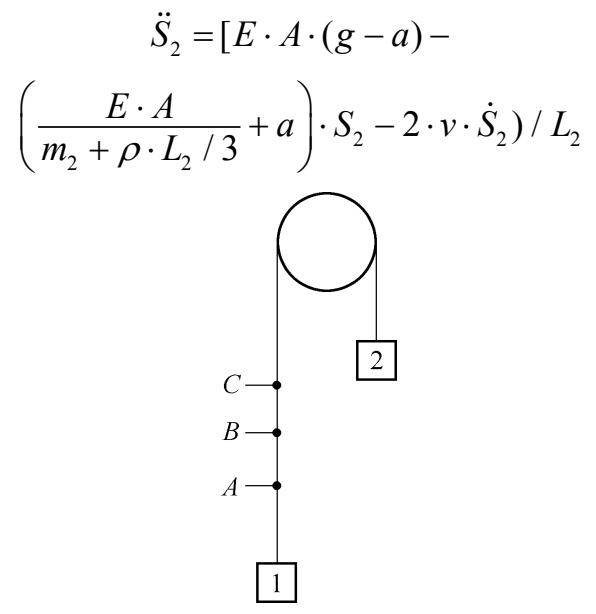

图 1 提升系统简图

上提侧、下降侧与摩擦轮切点处距离 $x$ 位置处 钢丝绳张力 $S_{1 x} 、 S_{2 x}$ 为(为简化计算, 忽略钢丝绳惯 性力影响, 较钢丝绳重量很小 $)^{[18]}$

$$
S_{1 x(2 x)}=S_{1(2)}-\rho x g
$$

对于弯曲段钢丝绳, 由于摩擦传动不可避免的 会发生弹性滑动, 因此围包角范围内包含滑移角 $\theta_{1}$ (钢丝绳和摩擦轮间存在滑移) 和静止角 $\theta_{2}$, 滑移角、 静止角及沿与摩擦轮接触区域的钢丝绳动张力为 ${ }^{[19]}$

$$
\begin{gathered}
F\left(\theta_{3}\right)=\left\{\begin{array}{cc}
S_{2} & 0<\theta_{3}<\theta_{2} \\
S_{2} \cdot \exp \left(m u\left(\theta_{3}-\theta_{2}\right)\right) & \theta_{2}<\theta_{3}<\pi
\end{array}\right. \\
\theta_{2}=\pi-\theta_{1} \\
\theta_{1}=\frac{1}{m u} \cdot \ln \frac{S_{1}}{S_{2}}
\end{gathered}
$$

式中, $E$ 为钢丝绳弹性模量; $A$ 为钢丝总断面积; $m_{1} 、 m_{2}$ 为终端质量; $g$ 为重力加速度; $a$ 为提升加(减) 速度; $\rho$ 为钢丝绳每米质量; $L_{1} 、 L_{2}$ 为上提与下放 侧容器距摩擦轮切点处距离; $v$ 为提升容器提升速 度; $S$ 为摩擦轮切点处钢丝绳张力; $S_{x}$ 为与摩擦轮 切点处距离 $x$ 处提升钢丝绳张力; $\theta_{1}$ 为滑移角; $\theta_{2}$ 为静止角; $m u$ 为钢丝绳与摩擦轮间摩擦因数; $F\left(\theta_{3}\right)$ 为摩擦轮圆心角 $\theta_{3}$ 处对应钢丝绳张力。

\section{2 内部钢丝拉力}

钢丝绳内部每根钢丝所受拉力为 ${ }^{[18]}$

$$
F_{k l}=\frac{\cos ^{2} \beta_{l} \cdot \cos ^{2} \alpha_{k l} \cdot E_{k l} \cdot A_{k l}}{\sum_{j=0}^{n_{s}}\left(z_{j} \cdot \cos ^{3} \beta_{j} \cdot \sum_{i=0}^{n_{k j}} z_{i j} \cdot \cos ^{3} \alpha_{i j} \cdot E_{i j} \cdot A_{i j}\right)} \cdot F
$$

绳股拉力等于股内所有钢丝在股轴线上拉力分量的 和 ${ }^{[18]}$

$$
F_{j}=\sum_{\mathrm{i}=0}^{n_{w j}} F_{i j} \cdot z_{i j} \cdot \cos \alpha_{i j}
$$

式中, $F$ 为钢丝绳所受拉力; $F_{k l}$ 为第 $l$ 股第 $k$ 丝层钢 
丝所受拉力; $\beta_{l}$ 为绳股捻角; $\alpha_{k l}$ 为第 $l$ 股 $k$ 丝层钢 丝捻角; $E_{k l}$ 为第 $l$ 股 $k$ 丝层钢丝弹性模量; $A_{k l}$ 为第 $l$ 股 $k$ 丝层钢丝横截面积; $n_{s}$ 为绳股层数; $j$ 为绳股层; $Z_{j}$ 为 $j$ 层绳股中绳股数; $\beta_{j}$ 为 $j$ 层绳股捻角; $n_{w j}$ 为 $j$ 层绳股中钢丝层数; $i$ 为钢丝层数; $Z_{i j}$ 为 $j$ 股 $i$ 丝层钢 丝数; $\alpha_{i j}$ 为 $j$ 股 $i$ 丝层钢丝捻角; $E_{i j}$ 为 $j$ 股 $i$ 丝层钢 丝弹性模量; $A_{i j}$ 为 $j$ 股 $i$ 丝层钢丝横截面积。

\section{3 内部钢丝相对滑移量}

在拉力作用下, 假设与容器连接端钢丝绳应变 为零, 则距离容器 $y$ 处钢丝伸长量为

$$
\begin{gathered}
\Delta l_{k l}=\frac{1}{E_{k l} \cdot A_{k l}} \cdot\left(S_{y} \cdot y+0.5 \cdot \rho \cdot g \cdot y^{2}\right) . \\
\sum_{j=0}^{n_{s}}\left(z_{j} \cdot \cos ^{3} \beta_{j} \cdot \sum_{i=0}^{n_{k j}} z_{i j} \cdot \cos ^{3} \alpha_{i j} \cdot E_{i j} \cdot A_{i j}\right)
\end{gathered}
$$

式中, $S_{y}$ 为距离容器 $y$ 处的钢丝绳张力; $\varepsilon_{k l}$ 为 $l$ 股 $k$ 丝层钢丝的应变。

拉力作用下钢丝绳内部捻制参数及钢丝间的相 对滑移计算公式见表 2。表中, $\Delta S P$ 为钢丝间相对

\section{表 2 钢丝绳内部捻制参数及相对滑移}

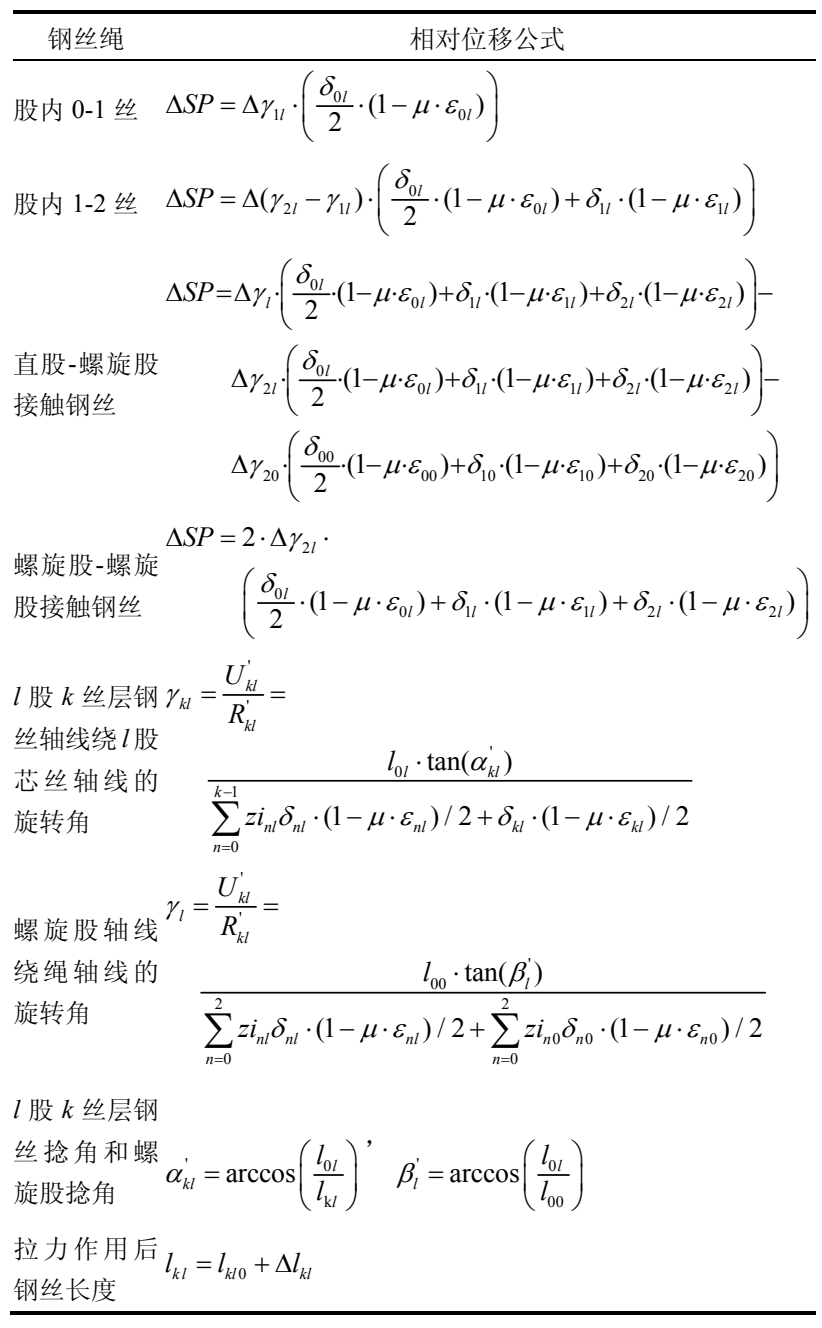

滑移; $\delta_{k l}$ 为 $l$ 股 $k$ 层钢丝直径; $l_{k l}$ 为 $l$ 股 $k$ 层钢丝 受力后长度; $l_{k l 0}$ 为 $l$ 股 $k$ 层钢丝未受力时长度; $\alpha_{k l}^{\prime}$ 为 $l$ 股 $k$ 层钢丝受力后捻角; $\beta_{l}^{\prime}$ 为 $l$ 股螺旋股受力 后捻角; $\gamma_{k l}$ 为 $l$ 股 $k$ 层钢丝旋转角; $U_{k l}^{\prime}$ 为 $l$ 股 $k$ 层钢丝缠绕弧长; $R_{k l}^{\prime}$ 为 $l$ 股 $k$ 层钢丝缠绕半径; $Z i_{n 1}$ 为沿钢丝绳径向 $l$ 股 $n$ 层钢丝数量; $\mu$ 为钢丝泊松比。

从文献 [20]可知, 提升过程中绳捻角变化值 $<0.63^{\circ}$, 股捻角的变化值 $<0.06^{\circ}$ 。因此, 本文忽略钢 丝绳的捻角变化, 以设定的捻角值代入计算。在计 算时, 先计算提升循环中各钢丝(绳股)缠绕弧长的 相对差值, 再计算相对滑移量的幅值。图 2 所示为 直股 1 层丝缠绕弧长 $U_{10}$, 其中曲线幅值即为直股 1 层丝与 0 层丝间相对滑移 $\Delta S P$ 。

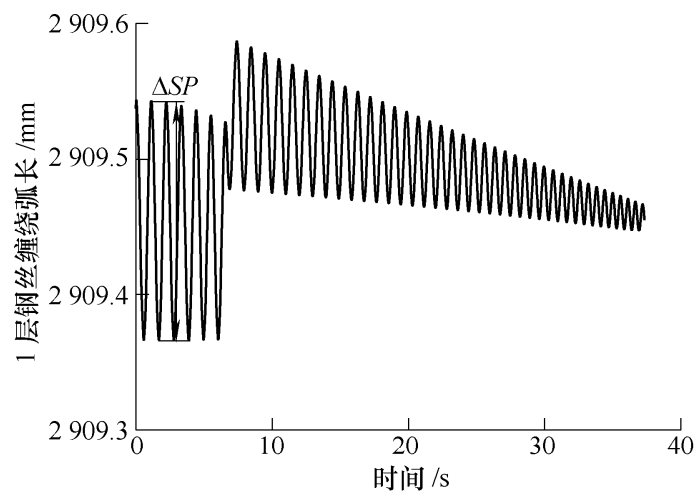

(a) 进入弯曲段前

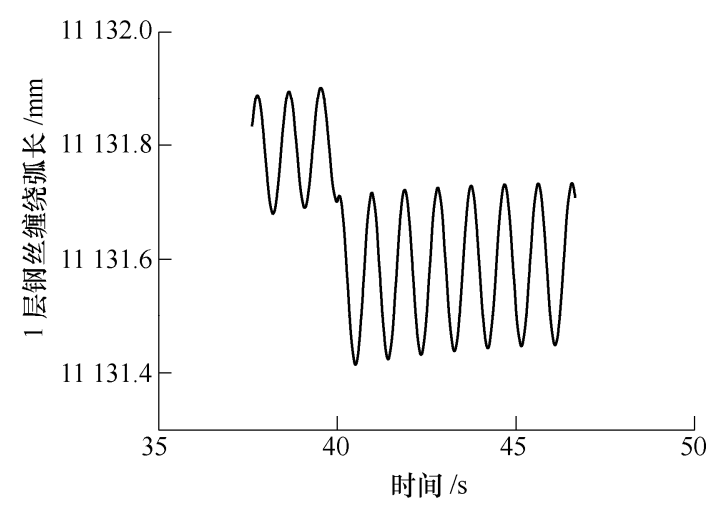

(b) 脱离弯曲段后

图 2 上提容器过程中距容器 $50 \mathrm{~m}$ 处钢丝绳直股内 1 层钢丝缠绕弧长

\section{4 内部钢丝接触载荷}

在拉力作用下, 股内相邻层间钢丝、直股-螺旋 股相邻钢丝、螺旋股-螺旋股相邻钢丝的接触载荷分 别为 ${ }^{[15]}$

$$
\begin{gathered}
P_{k(k+1)}=F_{k l} \cdot \sin \varphi \\
P=\frac{F_{s} \cdot \sin ^{2}\left(\beta_{1}\right) \cdot \pi \cdot \sin \left(\theta_{S}\right)}{Z_{s 2} \cdot \sin \left(\theta_{R}-\theta_{S}\right)} \\
P=\frac{F_{s} \cdot \sin ^{2}\left(\beta_{1}\right) \cdot L_{S}}{Z_{s 2} \cdot d_{C S}}
\end{gathered}
$$




$$
\theta_{S}=\arctan \left(\frac{L_{S}}{\pi \cdot d_{C S}}\right) \quad \theta_{R}=\arctan \left(\frac{L_{R}}{\pi \cdot d_{C S}}\right)
$$

式中, $P$ 为钢丝间接触载荷; $\alpha_{k l}$ 为第 $l$ 股第 $k$ 丝层 钢丝捻角; $\varphi$ 为松捻角, 其值与外层钢丝的捻角 $\alpha_{(k+1) l}$ 有关, 一般计算时, 当 $\alpha_{(k+1) l} \leqslant 6^{\circ}$ 时, $\varphi=60^{\circ}$; 当 $6^{\circ} \leqslant \alpha_{(k+1) l} \leqslant 12^{\circ}$ 时, $\varphi=50^{\circ}$; 当 $\alpha_{(k+1) l} \geqslant 12^{\circ}$ 时, $\varphi=40^{\circ} ; F_{s}$ 为螺旋股所受拉力; $\beta_{1}$ 为螺旋股捻角; $Z_{s 2}$ 为螺旋股最外层钢丝数; $L_{R}$ 为钢丝绳捻距; $L_{S}$ 为绳股捻距, $\mathrm{mm} ; d_{C S}$ 为直股外切圆直径。

图 3 为钢丝绳内部丝间接触载荷。由图 2 和 3 可知, 提升钢丝绳振动导致波动的钢丝间相对滑 移和变动的接触磨损区域, 根据对称性, 处于弯 曲段两侧时钢丝绳在拉力作用下相对滑移方向相 反, 文中假设摩擦轮同侧钢丝绳内部钢丝间接触 磨损区域的相对滑移中心位置不变, 取钢丝间接 触磨损区域相对滑移最小值(公共相对滑移区域) 作为钢丝间相对滑移, 以该最小值对应的钢丝间接 触载荷平均值作为钢丝间接触力。文献[18]认为弯 曲段钢丝绳捻角不变, 而且在提升循环中装载、卸 载以及钢丝绳弯曲时钢丝绳内部钢丝或股均分别
经历一次摩擦磨损, 相对于上提或下放过程中摩擦 磨损循环次数很小, 故本文仅考虑悬垂直线段钢丝 绳内部磨损。

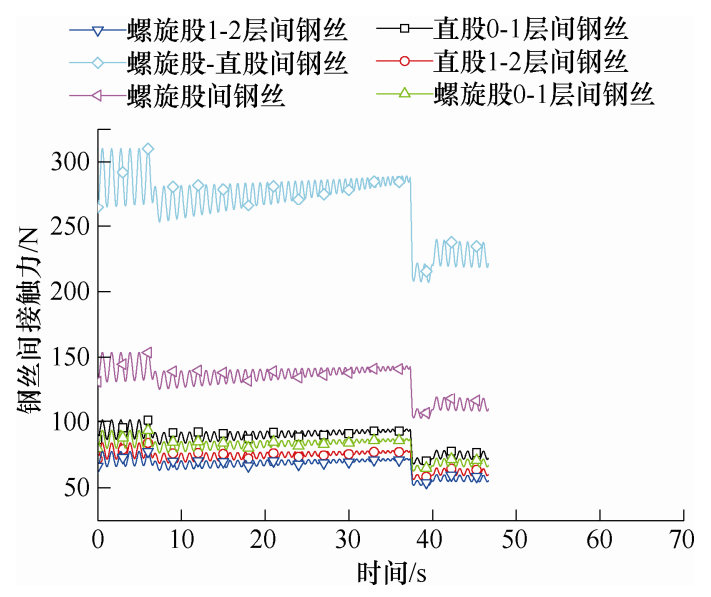

图 3 上提容器过程中距容器 $50 \mathrm{~m}$ 处钢丝绳内 钢丝间接触载荷

基于以上方法, 获得处于容器 1 侧上提(下放) 时钢丝绳微动磨损参数(表 3), 以接触力波动次数为 微动磨损循环次数。

表 3 上提(下放)时钢丝绳微动磨损参数

\begin{tabular}{|c|c|c|c|c|c|c|c|}
\hline \multirow{2}{*}{ 与容器 1 距离/m } & 运行 & \multirow{2}{*}{ 微动磨损参数 } & 直股 1-2 & \multirow{2}{*}{\multicolumn{2}{|c|}{$\begin{array}{c}\text { 螺旋股 1-2 } \\
\text { 层间钢丝 }\end{array}$}} & \multirow{2}{*}{$\begin{array}{c}\text { 螺旋股-直 } \\
\text { 股间钢丝 }\end{array}$} & \multirow{2}{*}{$\begin{array}{l}\text { 螺旋股 } \\
\text { 间钢丝 }\end{array}$} \\
\hline & 工况 & & 层间钢丝 & & & & \\
\hline \multirow{6}{*}{$50 \mathrm{~m}$} & \multirow{3}{*}{$\begin{array}{l}\text { 上提 } \\
\text { 重载 }\end{array}$} & 接触载荷/ N & 72 & 67 & & 267 & 132 \\
\hline & & 微动幅度/ mm & 0.0011 & 0.0011 & & 0.0379 & 0.0466 \\
\hline & & 循环次数 & \multicolumn{5}{|c|}{46} \\
\hline & \multirow{3}{*}{$\begin{array}{l}\text { 下放 } \\
\text { 空载 }\end{array}$} & 接触载荷/N & 50 & 46 & & 186 & 92 \\
\hline & & 微动幅度/ mm & 0.00006 & 0.00005 & & 0.01 & 0.0128 \\
\hline & & 循环次数 & \multicolumn{5}{|c|}{55} \\
\hline \multirow{6}{*}{$100 \mathrm{~m}$} & \multirow{3}{*}{$\begin{array}{l}\text { 上提 } \\
\text { 重载 }\end{array}$} & 接触载荷/N & 74 & 69 & \multirow{5}{*}{30} & 277 & 137 \\
\hline & & 微动幅度/mm & 0.0068 & 0.0063 & & 0.0777 & 0.0956 \\
\hline & & 循环次数 & & & & & \\
\hline & \multirow{3}{*}{$\begin{array}{l}\text { 下放 } \\
\text { 空载 }\end{array}$} & 接触载荷/ N & 53 & 49 & & 195 & 96 \\
\hline & & 微动幅度/ mm & 0.0001 & 0.0001 & & 0.0113 & 0.0146 \\
\hline & & 循环次数 & & & 36 & & \\
\hline \multirow{6}{*}{$150 \mathrm{~m}$} & \multirow{3}{*}{$\begin{array}{l}\text { 上提 } \\
\text { 重载 }\end{array}$} & 接触载荷/ N & 78 & 71 & \multirow{5}{*}{17} & 186 & 142 \\
\hline & & 微动幅度/mm & 0.00003 & 0.00003 & & 0.1195 & 0.1469 \\
\hline & & 循环次数 & & & & & \\
\hline & \multirow{3}{*}{$\begin{array}{l}\text { 下放 } \\
\text { 空载 }\end{array}$} & 接触载荷/ N & 56 & 51 & & 205 & 102 \\
\hline & & 微动幅度/ mm & 0.0002 & 0.0002 & & 0.0300 & 0.0385 \\
\hline & & 循环次数 & & & 32 & & \\
\hline
\end{tabular}

\section{2 提升钢丝绳内部钢丝微动磨损预测}

结合钢丝磨损实验数据, 构建不同损伤参数下 (交叉角、接触载荷、相对滑移)钢丝磨损演化模型, 进而获得一个提升循环中钢丝绳直径动态演化。

针对提升钢丝绳股间磨损情况, 因钢丝绳制造
原因(避免钢丝绳经过摩擦轮时外层螺旋股间挤压 和起棱而导致的钢丝绳寿命降低)和张力下绳股径 向收缩, 新钢丝绳螺旋股间存在间隙, 故直股一螺旋 股钢丝间首先发生微动磨损直至螺旋股一螺旋股钢 丝间发生接触; 因螺旋股一螺旋股的接触钢丝运动方 向不同, 故螺旋股一螺旋股钢丝间发生微动磨损; 当 螺旋股一螺旋股钢丝间磨损到一定程度后，直股一螺 
旋股钢丝间再度发生微动磨损过程。因此, 直股一 螺旋股、螺旋股-螺旋股的接触钢丝间交替发生微动 磨损现象, 为简化计算, 文中对直股-螺旋股或螺旋 股-螺旋股的钢丝间磨损系数均做减半处理。

图 $4 \mathrm{~b}$ 为图 $4 \mathrm{a}$ 中两接触钢丝在平面 $\mathrm{WP}$ (平面 $\mathrm{WP}$ 与移动钢丝轴线垂直)内的截面, 由图 $4 \mathrm{~b}$ 可知, 以一定角度交叉接触钢丝磨痕所在横截面分别为圆 形和椭圆形, 依据几何关系推导出两接触钢丝在横 截面 WP 上的总磨耗面积 $W A$ 与磨痕宽度 $b$ 的关联 关系为

$$
\begin{gathered}
W A=\frac{1}{4} \cdot \arcsin b-\frac{b}{4} \cdot \cos (\arcsin b)+ \\
\left(\frac{1}{4} \cdot \arcsin (b \cdot \cos \omega)-\right. \\
\left.\frac{b \cdot \cos \omega}{4} \cdot \cos (\arcsin (b \cdot \cos \omega))\right) / \cos \omega
\end{gathered}
$$

式中, $W A$ 为在横截面 $\mathrm{WP}$ 上两接触钢丝总磨耗 面积; $b$ 为移动钢丝磨痕宽度; $\omega$ 为接触钢丝交叉 捻角。

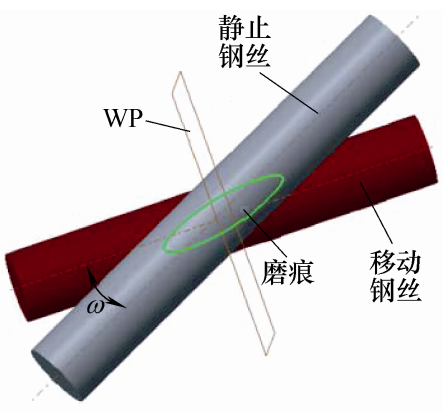

(a)

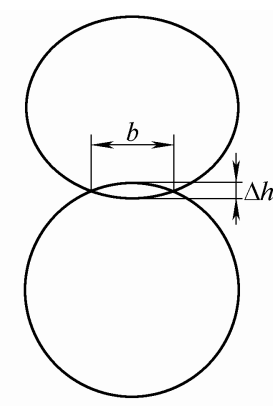

(b)
图 4 接触钢丝截面

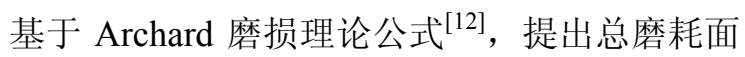
积 $W A$ 的理论预测模型

$$
\begin{gathered}
W A=k_{1} \cdot k_{2} \cdot k_{3} \cdot P \cdot \Delta S P \cdot \omega \cdot N= \\
k \cdot P \cdot \Delta S P \cdot \omega \cdot N
\end{gathered}
$$

式中, $k_{1} 、 k_{2} 、 k_{3}$ 分别为与接触载荷、相对滑移、交 叉捻角相关的磨损系数; $P$ 为接触载荷; $\Delta S P$ 为接 触钢丝间相对滑移; $N$ 为循环次数。

故总磨损系数 $k$ 为

$$
k=\frac{W A}{P \cdot \Delta S P \cdot \omega \cdot N}
$$

为了获取微动磨损计算参数, 设计了钢丝微 动磨损试验机(图 5), 步进电动机 1 和驱动滑台 2 实现往复循环移动, 激光位移传感器 6 实时测量 振幅, 导轨 3 上的加载平台 4 放置砝码, 向滑动 钢丝产生接触压力, 传感器 5 测量微动钢丝之间 的摩擦力。

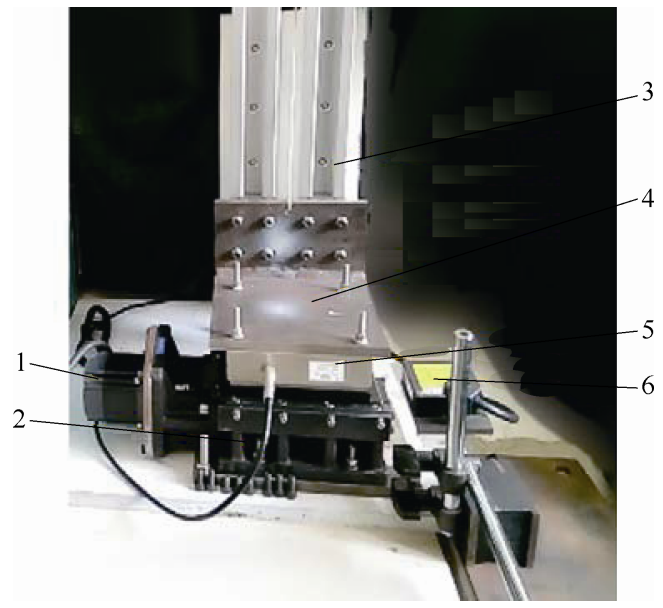

图 5 磨损试验机

钢丝固定夹具如图 6 所示, 为避免小交叉角时 夹具间相互干涉, 钢丝上夹具设计成图 6a 形式, 钢 丝嵌入夹具上的圆弧凹槽内, 通过夹具底座两侧的 螺钉进行固定。钢丝下夹具如图 $6 \mathrm{~b}$ 所示, 钢丝通过 两侧的压板进行固定。上夹具固定于传感器 5 上, 下夹具固定于滑台 2 上, 试验时两夹具相对位置如 图 6c 所示, 通过加工不同偏角的上夹具可实现不同 交叉捻角下的钢丝磨损试验。

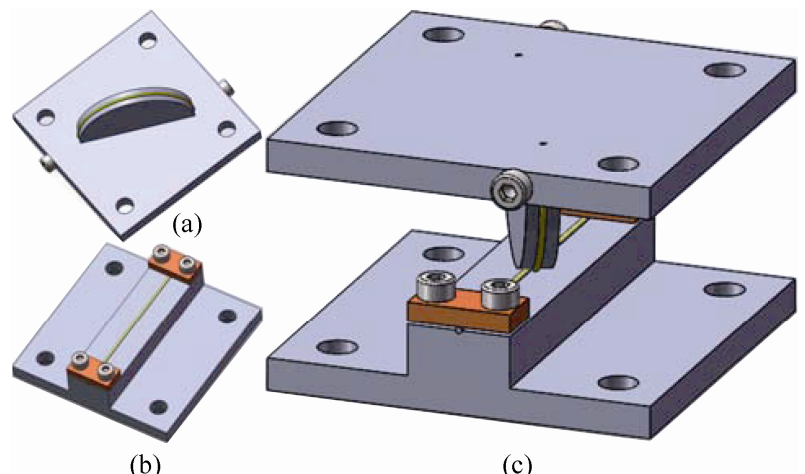

图 6 钢丝夹具

试验所用钢丝的表面均涂抹 IRIS-400M 矿山摩 擦提升机钢丝绳油脂(闪点 $\geqslant 35{ }^{\circ} \mathrm{C}$, 施油温度 0 $30{ }^{\circ} \mathrm{C}$ ), 风干 $1 \sim 2 \mathrm{~h}$ 后开始试验。

本文的钢丝微动磨损试验选取相对滑移为 100 $\mu \mathrm{m} 、 200 \mu \mathrm{m} 、 400 \mu \mathrm{m}$ 和 $800 \mu \mathrm{m}$, 接触载荷为 $10 \mathrm{~N}$ 、 $20 \mathrm{~N} 、 30 \mathrm{~N}$ 和 $40 \mathrm{~N}$, 具体的磨损循环次数、接触载 荷、往复滑移振幅、交叉捻角如表 4 所示。根据试 验测试的钢丝微动磨痕宽度, 由式(13)和式(15)求得 接触钢丝总磨损系数见表 4 。对比试验 $1 \sim 4$ 发现, 当循环次数超过 10000 次时, 已达到稳定磨损阶 段，总磨损系数就基本保持恒定。因此，本文试验 均以 10000 次微动作为总磨损系数确定的试验截止 次数。

为获得两接触钢丝总磨损系数预测模型, 分别 探究两接触钢丝总磨损系数随钢丝间接触载荷、相 
对滑移和交叉捻角演化特性, 开展钢丝摩擦磨损试 验。通过开展试验 5 8、9 12 和 13 15(表 4), 可以分别获得图 7 9 的关联关系。运用 Origin 软 件, 通过 ExpDec1、Slogistic1 和 Allometric1 模型分 别对图 7 9 中试验数据进行拟合(拟合度 $\mathrm{Adj}$. R-Square 分别为 $1.0 、 0.91 、 0.98)$, 分别获得两接触 钢丝总磨损系数与钢丝间接触载荷、相对滑移和交 叉捻角的关联关系

$$
\begin{gathered}
f_{p}=k_{1}(P) \cdot k_{2}(0.4) \cdot k_{3}(0.122)= \\
\eta \cdot \exp (-P / \lambda)+8.6965 \cdot 10^{-8} \\
f_{\Delta S P}=k_{1}(40) \cdot k_{2}(\Delta S P) \cdot k_{3}(0.122)= \\
\chi /(1+\exp (-\zeta \cdot(\Delta S P+0.17489))) \\
f_{\omega}=k_{1}(40) \cdot k_{2}(0.4) \cdot k_{3}(\omega)= \\
\left(7.05781 \cdot 10^{-8}\right) \cdot(\omega)^{-0.46053}
\end{gathered}
$$

\begin{tabular}{|c|c|c|c|c|c|c|}
\hline 序号 & $\begin{array}{l}\text { 相对 } \\
\text { 滑移 } \\
/ \mu \mathrm{m}\end{array}$ & $\begin{array}{l}\text { 接触 } \\
\text { 载荷 } \\
/ \mathrm{N}\end{array}$ & $\begin{array}{c}\text { 交叉角 } \\
/\left(^{\circ}\right)\end{array}$ & $\begin{array}{l}\text { 循环 } \\
\text { 次数/ } \\
\times 10^{3}\end{array}$ & $\begin{array}{l}\text { 磨痕 } \\
\text { 宽度 } \\
/ \mu \mathrm{m}\end{array}$ & $\begin{array}{c}\text { 磨损系数 } \\
/\left(\times 10^{-7} \mathrm{~mm} /\right. \\
(\mathrm{N} \mathrm{rad}))\end{array}$ \\
\hline 1 & 400 & 40 & 7 & 5 & 187.5 & 2.26 \\
\hline 2 & 400 & 40 & 7 & 10 & 222.22 & 1.89 \\
\hline 3 & 400 & 40 & 7 & 15 & 252.27 & 1.85 \\
\hline 4 & 400 & 40 & 7 & 20 & 279.55 & 1.89 \\
\hline 5 & 400 & 10 & 7 & 10 & 169.32 & 3.31 \\
\hline 6 & 400 & 20 & 7 & 10 & 198.86 & 2.69 \\
\hline 7 & 400 & 30 & 7 & 10 & 213.64 & 2.23 \\
\hline 8 & 400 & 40 & 7 & 10 & 222.22 & 1.89 \\
\hline 9 & 100 & 40 & 7 & 10 & 132.26 & 1.57 \\
\hline 10 & 200 & 40 & 7 & 10 & 169.32 & 1.66 \\
\hline 11 & 400 & 40 & 7 & 10 & 222.22 & 1.89 \\
\hline 12 & 800 & 40 & 7 & 10 & 281.82 & 1.94 \\
\hline 13 & 400 & 40 & 7 & 10 & 222.22 & 1.89 \\
\hline 14 & 400 & 40 & 10 & 10 & 234.09 & 1.53 \\
\hline 15 & 400 & 40 & 26 & 10 & 289.77 & 1.03 \\
\hline
\end{tabular}

式中, $\lambda 、 \eta 、 \chi 、 \xi$ 为拟合公式系数, $\lambda=34.15054$; $\eta=3.27718 \times 10^{-7} ; \chi=1.96953 \times 10^{-7} ; \xi=4.84075$ 。

表 4 试验参数工况及钢丝磨痕宽度

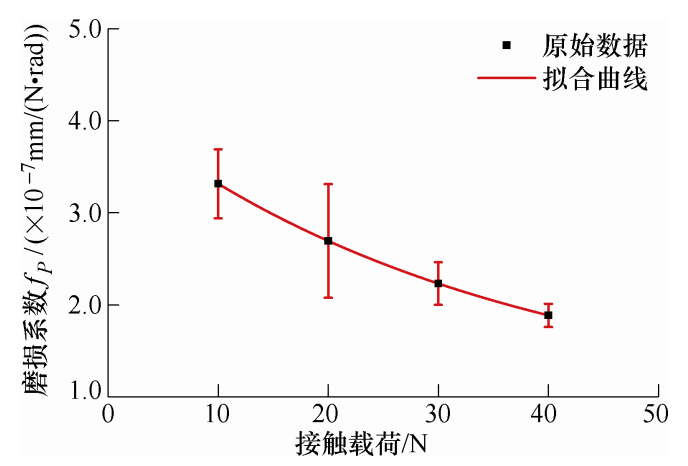

图 7 不同接触载荷下磨损系数演化
由式 $(16) \sim(18)$ 可得总磨损系数为

$$
\begin{gathered}
k=\frac{f_{p} f_{\Delta S P} f_{\omega}}{\left(k_{1}(40) \cdot k_{2}(0.4) \cdot k_{3}(0.122)\right)^{2}}= \\
k_{1}(P) \cdot k_{2}(\Delta S P) \cdot k_{3}(\omega)
\end{gathered}
$$

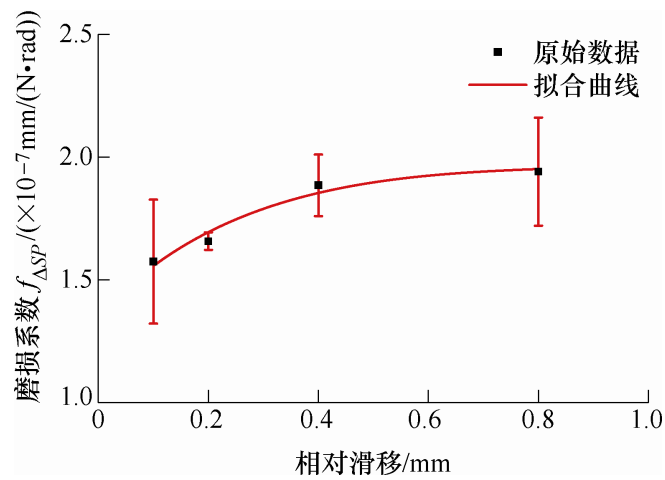

图 8 不同相对滑移下磨损系数演化

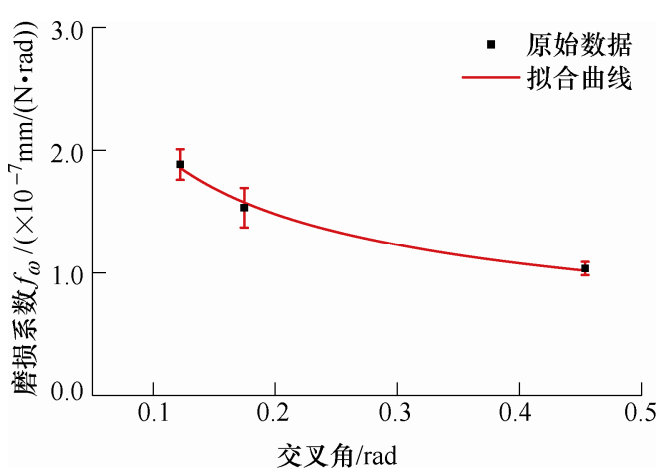

图 9 不同交叉角下磨损系数演化

\section{3 提升钢丝绳承载安全系数预测}

\section{1 内部钢丝磨耗总面积变化}

结合表 1 提升工况参数、第 2 节提升循环中钢 丝绳微动损伤参数以及式(14)和(19), 可获得一次提 升循环中 (上提和下放过程)距容器不同位置处钢丝 绳内部钢丝总磨损磨耗面积(图 10)。由此可见, 直

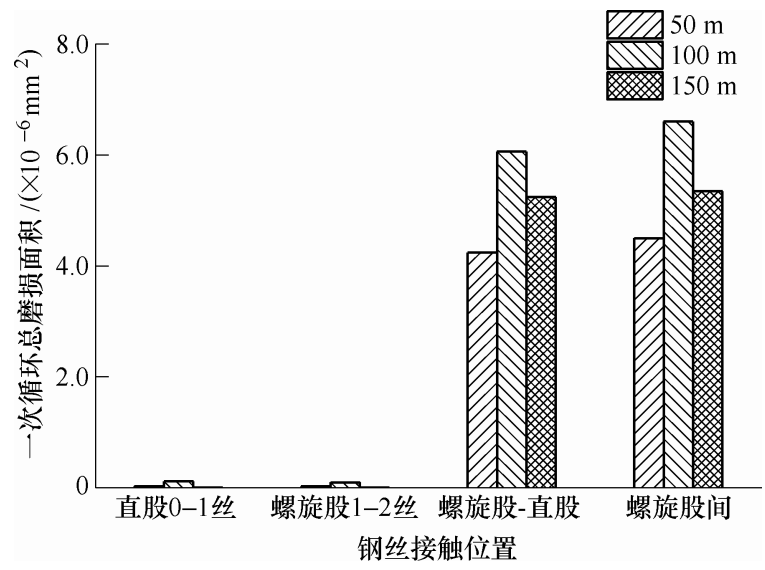

图 10 一次提升循环中距离容器不同位置处钢丝绳 内部钢丝磨损磨耗总面积 
股-螺旋股、螺旋股-螺旋股的接触钢丝磨耗面积较 大，而股内层间接触钢丝磨损磨耗面积很小，这 是由于股内钢丝间接触力和相对滑移较小引起 的。根据《煤矿安全规程》规定, 钢丝绳断丝减 少横截面积达到 $10 \%$, 即横截面积减少 10.45 $\mathrm{mm}^{2}$ (钢丝绳初始横截面积为 $104.5 \mathrm{~mm}^{2}$ ) 时钢丝绳 报废(为简化计算, 文中不考虑钢丝绳内部钢丝疲 劳断裂情况)，报废线见图 11。《煤矿安全规程》 规定提升钢丝绳使用期限应不超过 2 年(24 个月), 如果钢丝绳未达到报废标准, 可继续使用但不超 过 1 年(12月)。

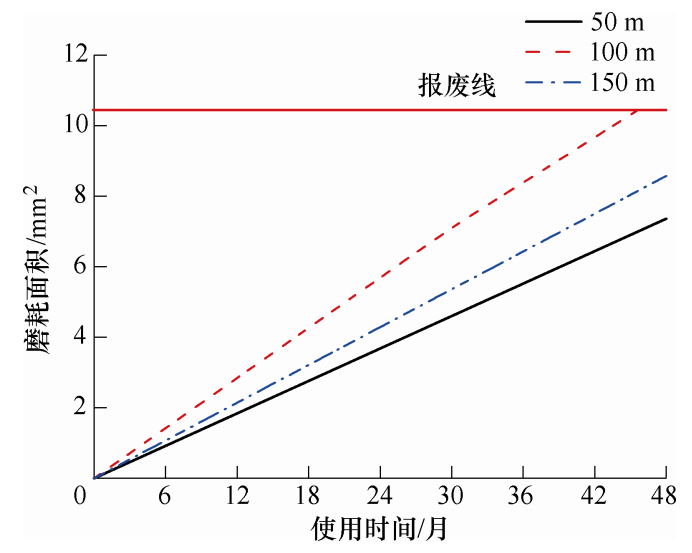

图 11 距离容器不同位置处钢丝绳动态磨损演化

针对本文选择的矿井提升工况, 以每年工作 300 天，净提升时间 15 小时/天，30 次/小时 $(15$ 个提升循环(小时); 若每月平均工作 25 天, 则钢 丝绳提升循环次数可转化为图 11 所示使用月数。 可见, 提升钢丝绳使用期限达到 2 年时距容器不 同三个位置处钢丝绳均未达到报废标准, 而距容 器 $100 \mathrm{~m}$ 处钢丝绳在使用到约 45 个月时达到报废 标准。

\section{2 钢丝绳承载安全系数变化}

目前钢丝绳的选型设计都采用静强度设计理 论, 即以静态破断极限为基础, 增大安全系数来 确定钢丝绳的许用承载能力。然而, 这种方法无 法对服役钢丝绳的安全状态做出准确预测和评 估, 因而会出现在规定服役期内没有失效而提前 更换报废钢丝绳的 “过度安全” 情况, 或者未到 服役期就发生钢丝绳断裂失效事故的危险情况。

因此根据《煤矿安全规程》, 提出钢丝绳承载安全 系数见式(20)。

$$
S_{\mathrm{a}}=\frac{(A-\Delta A) \cdot S_{0}}{A}
$$

式中, $S_{a}$ 为提升钢丝绳承载安全系数; $S_{0}$ 为提升 钢丝绳初始安全系数, 依据《煤矿安全规程》取
$10 ; A$ 为钢丝绳横截面积, $\mathrm{mm}^{2} ; \Delta A$ 为钢丝绳磨 耗面积。

随着提升循环次数的增加, 距容器不同位置处 钢丝绳内部钢丝磨耗总面积递增(图 11), 钢丝绳实 际承载截面积减小，由式(20)可得钢丝绳实际承载 安全系数呈动态降低趋势, 如图 12 所示, 钢丝绳达 到报废标准时安全系数为 9。可见，中间位置处钢 丝绳承载安全系数下降速率较大，钢丝绳达到报废 时间较短。《煤矿安全规程》规定摩擦式提升机提升 钢丝绳更换年限为 2 年，本文所预测的钢丝绳承载 安全系数在约 45 个月时达到报废标准, 因此本文承 载安全系数变化相对于《煤矿安全章程》规定的报 废年限留有安全裕度。

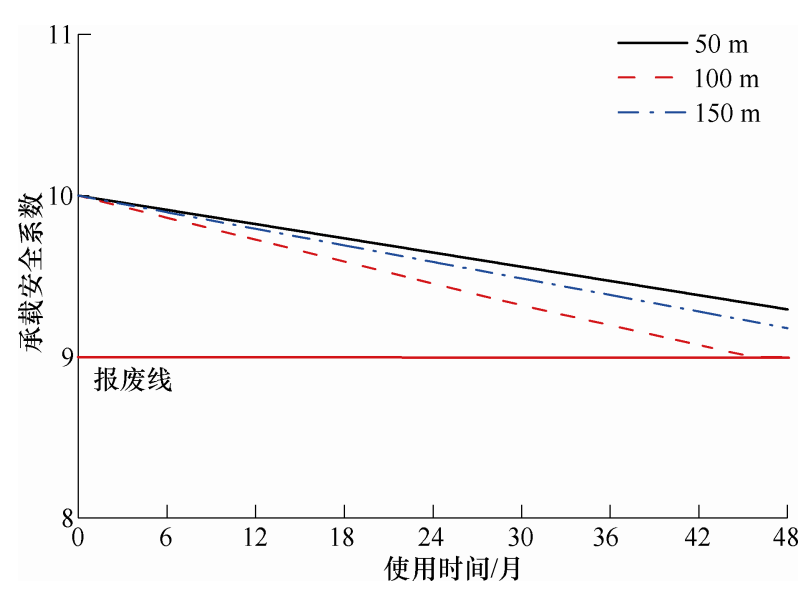

图 12 距离容器不同位置处钢丝绳承载安全系数演化

\section{3 运行参数对钢丝绳承载安全系数的影响}

本文选取距容器 $50 \mathrm{~m}$ 位置进行不同工况下的 钢丝绳承载安全系数研究, 其微动磨损参数计算结 果见表 5。随着提升侧终端质量的增加, 钢丝绳磨 损速率增加, 承载安全系数下降加快, 如图 13 所示; 随着最大提升速度的增加, 钢丝绳磨损速率降低, 承载安全系数下降减慢, 如图 14 所示。

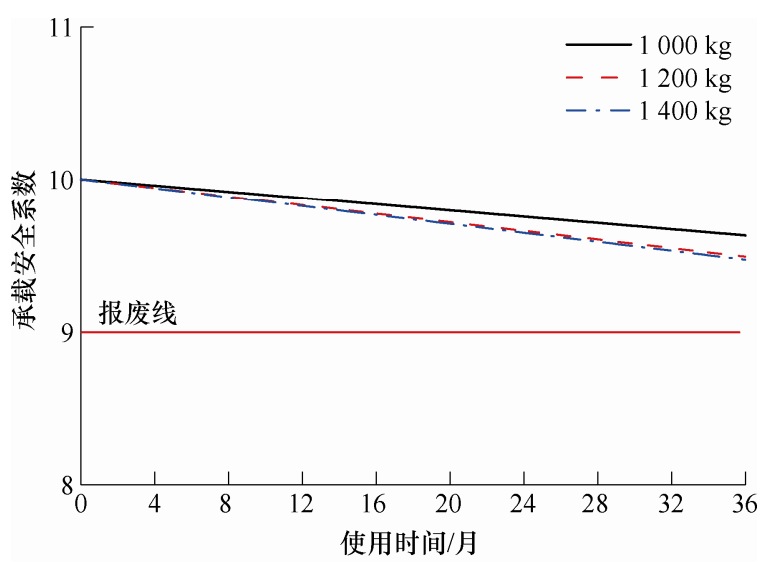

图 13 提升载荷变化对钢丝绳承载安全系数的影响 


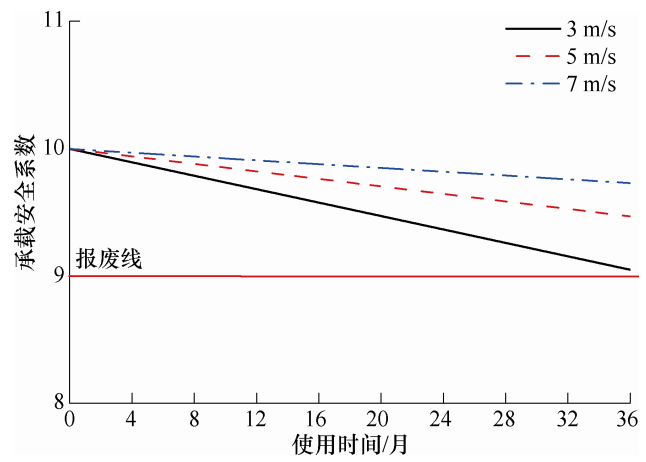

图 14 最大提升速度变化对钢丝绳承载安全系数的影响
提升侧终端质量增大使钢丝间接触载荷和相对 滑移增大，因而钢丝绳磨损速率及承载安全系数下 降速度增加。提升速度增大使一个提升循环的内部 钢丝经历的微动磨损循环次数减小，表明提升速度 的增加导致提升周期缩短，进而导致钢丝间微动磨 损循环次数的明显减小。由于微动磨损循坏次数对 钢丝绳微动磨损的影响比接触载荷与相对滑移的影 响更为显著，最终使钢丝绳磨损速率及承载安全系 数下降速度减小。

表 5 不同运行工况下的 $50 \mathrm{~m}$ 处钢丝绳内部钢丝或股的微动磨损参数

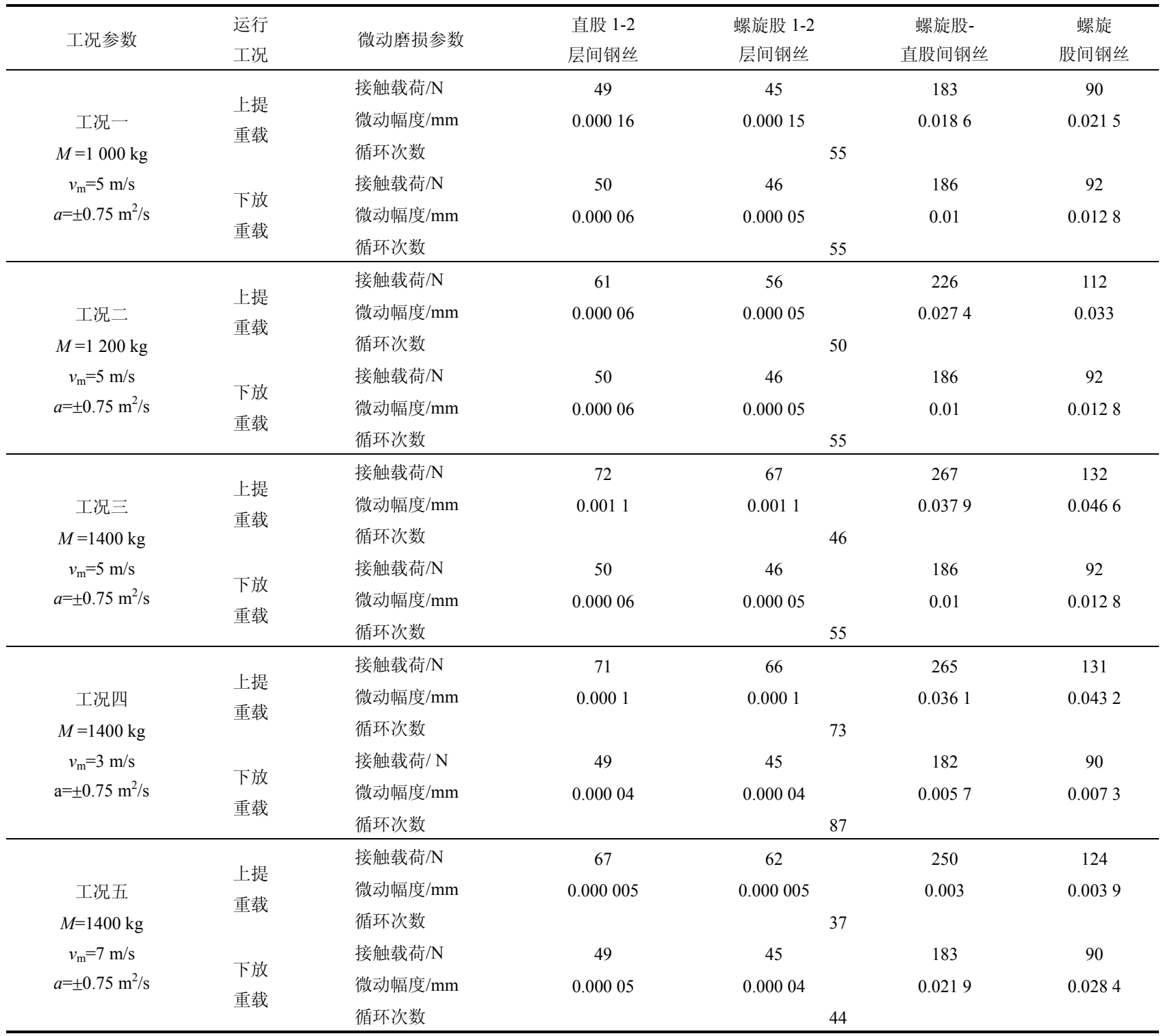

\section{4 结论}

本文提出了矿井提升钢丝绳承载安全系数预测 方法, 基于浅井低载荷矿井提升机研究发现, 提升 钢丝绳因为在运行过程中发生微动磨损而使其承载 安全系数随之降低。钢丝绳悬垂中间位置处钢丝绳
承载安全系数下降较快, 在使用约 45 个月时钢丝绳 承载安全系数才会达到现行《煤矿安全章程》的报 废标准, 远超过规定的 2 年更换年限。此外, 提升 钢丝绳的承载安全系数对提升工况参数具有较大敏 感性, 提升终端质量的增加导致钢丝绳承载安全系 数下降, 最大提升速度的减小导致承载安全系数下 降。本文提出的理论模型对实现提升钢丝绳安全寿 
命设计具有重要的理论意义。

\section{参 考 文 献}

[1] 张德坤, 葛世荣, 熊党生. 矿井提升机用提升钢丝绳的微 动磨损行为研究 [J]. 摩擦学学报, 2001, 21(5): 362-365. ZHANG Dekun, GE Shirong, XIONG Dangshen. Fretting wear behavior of hoisting rope in mining hoister[J]. Tribology, 2001, 21(5): 362-365.

[2] 马军, 葛世荣, 张德坤. 钢丝绳股内钢丝应力一应变分 布的计算模型及数值模拟[J]. 机械工程学报, 2009, 45(11): 277-282.

MA Jun, GE Shirong, ZHANG Dekun. Calculating model and numerical simulation of stress-strain distribution of wires within strands[J]. Journal of Mechanical Engineering, 2009, 45(11): 277-282.

[3] 马军, 葛世荣, 张德坤. 钢丝绳应力及股内钢丝变形的 相关性研究[J]. 煤炭科学技术, 2009, 37(3): 73-76.

MA Jun, GE Shirong, ZHANG Dekun. Research on relativity between steel cable stress and steel wire deformation of strand[J]. Coal Science and Technology, 2009, 37(3): 73-76.

[4] WANG D, ZHANG D, WANG S, et al. Finite element analysis of hoisting rope and fretting wear evolution and fatigue life estimation of steel wires[J]. Engineering Failure Analysis, 2013，27: 173-193.

[5] 张德坤, 葛世荣, 朱真才. 提升钢丝绳的钢丝微动摩擦磨 损特性研究[J]. 中国矿业大学学报, 2002, 31(5): 367-370. ZHANG Dekun, GE Shirong, ZHU Zhencai. Friction and wear performance on fretting wear of steel wires in hoisting ropes[J]. Journal of China University of Mining\&Technology, 2002, 31(5): 367-370.

[6] 张德坤, 葛世荣. 钢丝微动磨损的评定参数及理论模型 研究 $[J]$. 摩擦学学报, 2005, 25(1): 50-54.

ZHANG Dekun, GE Shirong. Research on the evaluation parameters and theory model of fretting wear between steel wires[J]. Tribology, 2005, 25(1): 50-54.

[7] 张德坤, 葛世荣. 钢丝微动磨损过程中的接触力学问题 研究 $[J]$. 机械强度, 2007，29(1)：148-151。

ZHANG Dekun, GE Shirong. Research on the contact mechanisms in the process of fretting wear between steel wires[J]. Journal of Mechanical Strength，2007，29(1): 148-151.

[8] 沈燕, 张德坤, 王大刚, 等. 接触载荷对钢丝微动磨损 行为影响的研究 [J]. 摩擦学学报, 2010, 30(4): 404-408. SHEN Yan, ZHANG Dekun, WANG Dangang, et al. Effect of contact load on the fretting wear behavior of steel wire[J]. Tribology, 2007，29(1): 148-151.

[9] 张德坤, 葛世荣, 朱真才. 评定钢丝的微动摩擦磨损参 数研究 $[J]$. 中国矿业大学学报, 2004, 33(1): 33-36. ZHANG Dekun, GE Shirong, ZHU Zhencai. Research on evaluation of fretting parameters of steel wires[J]. Journal of China University of Mining\&Technology, 2004, 33(1): 33-36.

[10] 殷艳, 张德坤, 沈燕. 润滑油脂对钢丝微动磨损特性的 影响[J]. 摩擦学学报, 2011, 31(5): 492-497.

YIN Yan, ZHANG Dekun, SHEN Yan. Effect of grease lubrication on the fretting wear properties of steel wires[J]. Tribology, 2011, 31(5): 492-497.

[11] 张德坤, 葛世荣. 提升钢丝绳的微动损伤试验研究 [J]. 矿山机械, 1998(3): 47-48.

ZHANG Dekun, GE Shirong. Experimental research on the fretting wear of hoisting rope[J]. Mining\&Processing Equipment, 1998(3): 47-48.

[12] CRUZADO A, HARTELT M, WÄSCHE R, et al. Fretting wear of thin steel wires. Part 1: Influence of contact pressure[J]. Wear, 2010, 268(11): 1409-1416.

[13] CRUZADO A, HARTELT M, WÄSCHE R, et al. Fretting wear of thin steel wires. Part 2: Influence of crossing angle[J]. Wear, 2011, 273(1): 60-69.

[14] WANG D, ZHANG D, ZHAO W, et al. Quantitative analyses of fretting fatigue damages of mine rope wires in different corrosive media[J]. Materials Science and Engineering: A, 2014, 596: 80-88.

[15] WANG D, ZHANG D, GE S. Effect of terminal mass on fretting and fatigue parameters of a hoisting rope during a lifting cycle in coal mine[J]. Engineering Failure Analysis, 2014, 36: 407-422.

[16] 严世榕, 闻邦椿. 提升钢丝绳张力的计算机仿真[J]. 煤 矿机械, 1998(3): 4-6.

YAN Shirong, WEN Bangcun. Computer simulation of rope tension during the lifting process[J]. Coal Mine Machinery, 1998(3): 4-6.

[17] 严世榕, 闻邦椿. 下放容器时提升钢丝绳的动力学仿真 [J]. 煤炭学报, 1998, 23(5): 530-534.

YAN Shirong, WEN Bangcun. Dynamic simulation of hoisting rope during the lowering process[J]. Journal of China Coal Society, 1998, 23(5): 530-534.

[18] FEYRER K. Wire ropes[M]. Berlin: Springer-Verlag Berlin Heidelberg, 2007.

[19] WANG D, ZHANG D, MAO X, et al. Dynamic friction transmission and creep characteristics between hoisting rope and friction lining $[\mathrm{J}]$. Engineering Failure Analysis, 2015, 57: 499-510.

[20] ZHANG J, WANG D, ZHANG D, et al. Dynamic torsional characteristics of mine hoisting rope and its internal spiral components[J]. Tribology International, 2017, 109: 182-191.

作者简介: 张俊, 男, 1994 年出生。主要研究微动磨损。

E-mail: zhangjun@cumt.edu.cn

葛世荣(通信作者), 男, 1963 年出生, 博士, 教授, 博士研究生导师。 主要研究摩擦学与可靠性、矿山机器人等。

E-mail: gesr@cumt.edu.cn 